\title{
Multilevel Coding in Free-Space Optical MIMO Transmission With $Q$-Ary PPM Over the Atmospheric Turbulence Channel
}

\author{
Ivan B. Djordjevic, Bane Vasic, and Mark A. Neifeld
}

\begin{abstract}
We propose an efficient coded modulation scheme for multiple input/multiple output free-space optical systems with $Q$-ary pulse-position modulation. It is based on multilevel coding and low-density parity-check codes as component codes. The proposed scheme provides excellent coding gain in the regime of strong atmospheric turbulence.
\end{abstract}

Index Terms-Atmospheric turbulence channel, free-space optics, multilevel coding (MLC), multiple input/multiple output (MIMO) processing, pulse-position modulation (PPM).

\section{INTRODUCTION}

C UURENT free-space optical (FSO) communication systems [1]-[3] employ intensity modulation with direct detection (IM/DD) and use point-to-point communication between two optical transceivers along a line of sight [1]. FSO communication is an attractive and cost-effective solution for high-rate image, voice, and data transmission [1]-[3]. However, an optical wave propagating through the air experiences fluctuations in amplitude and phase due to atmospheric turbulence [4]. The effect is known as scintillation. It is one of the most important factors that degrade the performance of an FSO communication link, even under the clear sky conditions. An effective way to overcome this problem is to use multiple lasers and multiple photodectors [2], [3] in a fashion analogous to multiple input/multiple output (MIMO) wireless communications. The laser sources and photodetectors are positioned in such a way that intensity fluctuations are statistically independent between any source-detector pair [2], [3]. Previous studies [8] have shown that the performance of an FSO system is degraded most severely by deep fog because of considerable signal attenuation. To increase transmission distance higher laser powers or alternatively power efficient modulation schemes are necessary. The schemes such as pulse amplitude modulation (PAM) and quadrature amplitude modulation (QAM) that are commonly used in wireless communications provide high spectral efficiency but are not power efficient and are, therefore, not a good choice. The $Q$-ary pulse-position modulation (PPM) is an attractive power-efficient modulation scheme for FSO links [2], [3], [5]. The large bandwidth of FSO links (compared to RF links) makes the low spectral efficiency of PPM of little concern [8]. Moreover, as the number of slots in $Q$-ary PPM increases, the influence of background radiation becomes less important [2], [3], because the photodiode integration interval

Manuscript received April 10, 2006; revised April 27, 2006. This work was supported in part by the National Science Foundation under Grant ITR 0325979.

The authors are with the Department of Electrical and Computer Engineering, University of Arizona, Tucson, AZ 85721 USA (e-mail: ivan@ece.arizona.edu).

Digital Object Identifier 10.1109/LPT.2006.877576

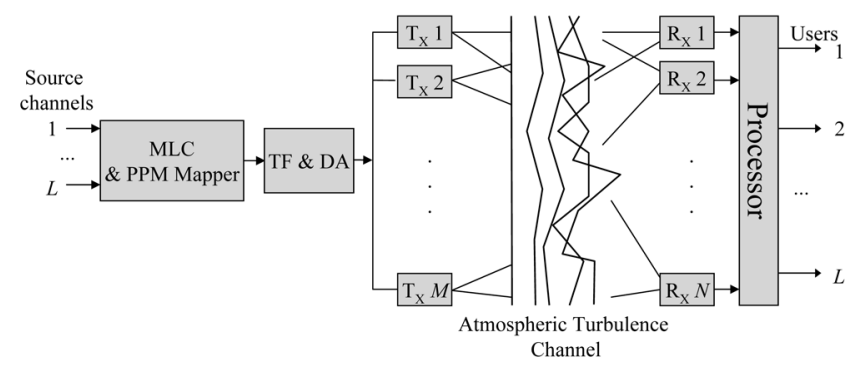

Fig. 1. Atmospheric optical MIMO system with $Q$-ary PPM and MLC. TF: transmitter filter. DA: driver amplifier. $\mathrm{T}_{\mathrm{X}}$ : transmitter. $\mathrm{R}_{\mathrm{X}}$ : receiver. (Color version available online at http://ieeexplore.iee.org.)

is shorter. To improve the performance of FSO systems with PPM, several forward error correction (FEC) schemes have been proposed, including RS codes [6], convolutional codes [7], trellis-coded modulation (TCM) [5], and turbo codes [8]. Coding gains of RS and convolutional codes are sufficient under the regime of weak turbulence and in the absence of fog. However, in the presence of strong turbulence or deep fog, coding gains of RS or convolutional codes are insufficient, as shown in [10], and more advanced FEC schemes, such as turbo [8] or low-density parity-check (LDPC) codes [10], are needed.

In this letter, we propose a power efficient coded-modulation scheme based on multilevel coding (MLC) [9] with LDPC codes [10] as component codes, suitable for the use in repetition MIMO FSO systems with $Q$-ary PPM. The proposed coded modulation scheme allows aggregation of $\mathrm{RF} /$ microwave signals and a conversion to the optical domain in a very natural way and may be a good candidate for hybrid RF/microwave-FSO systems. $L=\log _{2} Q$ bit streams from $L$ independent RF channels are independently coded using $L$ LDPC codes and converted to a light pulse position using $Q$-ary PPM signaling. The MLC scheme considered in this letter provides excellent coding gains, and unlike the previously reported methods [5]-[8], it is able to operate in the strong atmospheric turbulence regime. The bit-error rate (BER) performance is assessed assuming an ideal photon counting receiver, in the presence of background radiation. The influence of scintillation is modeled assuming a Gamma-Gamma distribution [4].

\section{SYSTEM ARCHITECTURE}

A block diagram of the proposed coded-modulation repetition MIMO $Q$-ary PPM FSO communication system is given in Fig. 1. The encoder and decoder configurations are given in Fig. 2(a) and (b), respectively. A $Q$-ary PPM scheme transmits $L=\log _{2} Q$ bits per symbol, providing high power efficiency. The source bit streams coming from $L$ sources $u_{i}(i=$ 


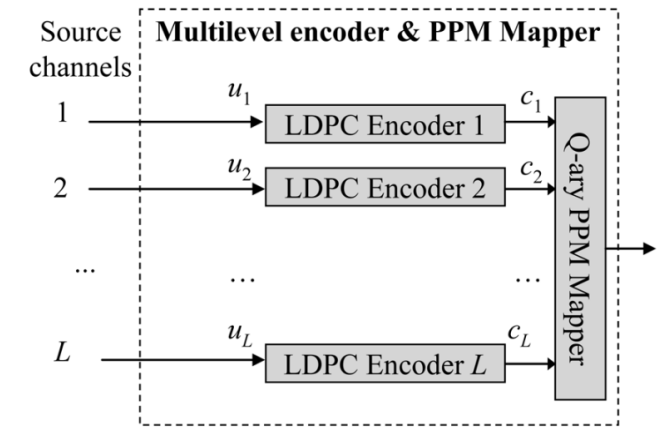

(a)

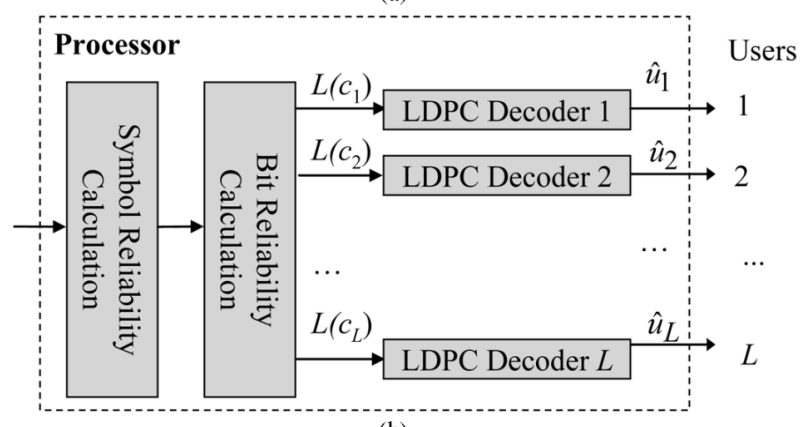

(b)

Fig. 2. Block schemes of (a) multilevel encoder and $Q$-ary PPM mapper and (b) parallel independent decoders. (Color version available online at http://ieeexplore.ieee.org.)

$1,2, \ldots, L)$ are encoded using $L\left(n, k_{i}\right)$ LDPC encoders of rate $R_{i}=k_{i} / n$, where $n$ is the codeword length and $k_{i}$ is the dimension (information word length) of the $i$ th component LDPC code. The source bits may originate from a single source as well. In that case, a serial-to-parallel (1-to- $L$ ) converter is required. The signaling interval $T_{s}$ is subdivided into $Q$ slots of duration $T=T_{s} / Q$. The natural mapping device maps a binary vector $\boldsymbol{c}=\left(c_{1}, c_{2}, \ldots, c_{L}\right)$ into one of $Q=2^{L}$ time slots. With proper arrangement of information bits within an LDPC codeword or with interleaving, any $L$ neighboring information bits, upon encoding, may be allocated into different PPM symbols. This technique improves the tolerance to atmospheric turbulence, because different $Q$-ary PPM symbols exhibit different atmospheric turbulence conditions. At the receiver side, LDPC decoders operate independently and in parallel. Because the inherently large delay of the multistage decoding algorithm is not acceptable in high-speed applications, our scheme employs a simplified multistage decoding known as parallel independent decoding (PID) [11].

In each signaling interval $T_{s}$, a pulse of light of duration $T$ is transmitted by a laser. For data rates below 10 Gsymbols/s the direct modulated laser is employed, and for data rates of 10 Gsymbols/s and above an external Mach-Zehnder modulator and distributed-feedback laser (DFB) are employed instead. To achieve the repetition MIMO transmission $M$ laser sources and $N$ photodetectors (PDs) are employed. The transmitted power per laser is denoted by $P_{\text {tot }} / M$, and received power (after losses) per PD is $P_{r} . P_{\text {tot }}$ is the total transmitted power, which is fixed and independent of the number of lasers being employed. The spectral efficiency can be calculated as $S=R_{s} /\left(Q / T_{s}\right)$, where the number of bits per symbol $R_{s}$ is determined as the sum of the individual code rates $R_{i}=k_{i} / n$ [11]

$$
R_{s}=\sum_{i=0}^{L-1} R_{i}=\frac{1}{n} \sum_{i=0}^{L-1} k_{i}=\frac{k}{n}, \quad k=\sum_{i=0}^{L-1} k_{i} .
$$

The received optical energy per uncoded symbol (per one PD) $E_{s}$ is determined by $E_{s}=P_{r} T=E_{b} \log _{2} Q$, where $E_{b}$ is the energy per information bit. The $i$ th $(i=1,2, \ldots, M)$ transmitter modulated beam is projected toward the $j$ th $(j=$ $1,2, \ldots, N)$ receiver using the expanding telescope. At the receiver, an optical system collects the light and focuses it onto a detector, which delivers an electrical signal proportional to the incoming optical power. The receiver commonly employs the transimpedance design, which is a good compromise between noise and bandwidth. A preamplified PIN photodiode or an avalanche photodiode (APD) are typically used as optical detectors. The PIN PD is employed here. During propagation through the air, the optical beam experiences amplitude and phase variations caused by scattering, refraction caused by atmospheric turbulence, absorption, and building sway.

In a receiver, all LDPC decoders operate independently and in parallel. The LDPC decoders input reliabilities $L\left(c_{j}\right)$ are calculated from the symbol reliabilities $\lambda(q)(q=1,2, \ldots, Q)[q$ th symbol corresponds to $\left.\mathbf{c}=\left(c_{1}, c_{2}, \ldots, c_{L}\right)\right]$ as

$$
L\left(c_{j}\right)=\log \frac{\sum_{\mathbf{c}: c_{j}=0} \exp [\lambda(q)]}{\sum_{\mathbf{c}: c_{j}=1} \exp [\lambda(q)]}
$$

while the symbol reliabilities are calculated, as explained in [2], by

$$
\lambda(q)=\sum_{n=1}^{N} Z_{n q} \log \left(\frac{\frac{n_{s}}{M} \sum_{m=1}^{M} I_{n m}+n_{b}}{n_{b}}\right) .
$$

The number of photoelectrons in the $q$ th slot of $n$th PD, in which a pulse is sent, denoted as $Z_{n q}$, has a Poisson count distribution [2] with the mean parameter $\left(n_{s} / M\right) \sum I_{n m}+n_{b} . I_{n m}$ is the intensity of the light incident to $n$th PD, originated from $m$ th laser source. The slot without a pulse has a Poisson distribution with mean parameter $n_{b}$. The PD analysis is based on a semiclassical treatment of the photodetection process as explained in [2]. $n_{s}$ corresponds to the PD effective number of photoelectrons per slot (per one PD) in the absence of turbulence and background light [2]

$$
n_{s}=\frac{\eta P_{r} T}{h(c / \lambda)}=\frac{\eta E_{s}}{h(c / \lambda)} \quad \text { [photoelectrons/slot] }
$$

while $n_{b}$ corresponds to the effective number of photoelectrons due to background light

$$
n_{b}=\frac{\eta P_{b} T}{h(c / \lambda)} \quad[\text { photoelectrons/slot }]
$$

where $P_{b}$ is the PD incident background noise power, $\eta$ is the photodiode efficiency, set in simulations to $0.5, h$ is the Plank constant, $c$ is the speed of the light in vacuum, and $\lambda$ is the wavelength, set in simulations to $1550 \mathrm{~nm}$. The influence of the atmospheric turbulence channel is described by the Gamma-Gamma probability density function (PDF) [4]

$$
f(I)=\frac{2(\alpha \beta)^{(\alpha+\beta) / 2}}{\Gamma(\alpha) \Gamma(\beta)} I^{(\alpha+\beta) / 2-1} K_{\alpha-\beta}(2 \sqrt{\alpha \beta I})
$$




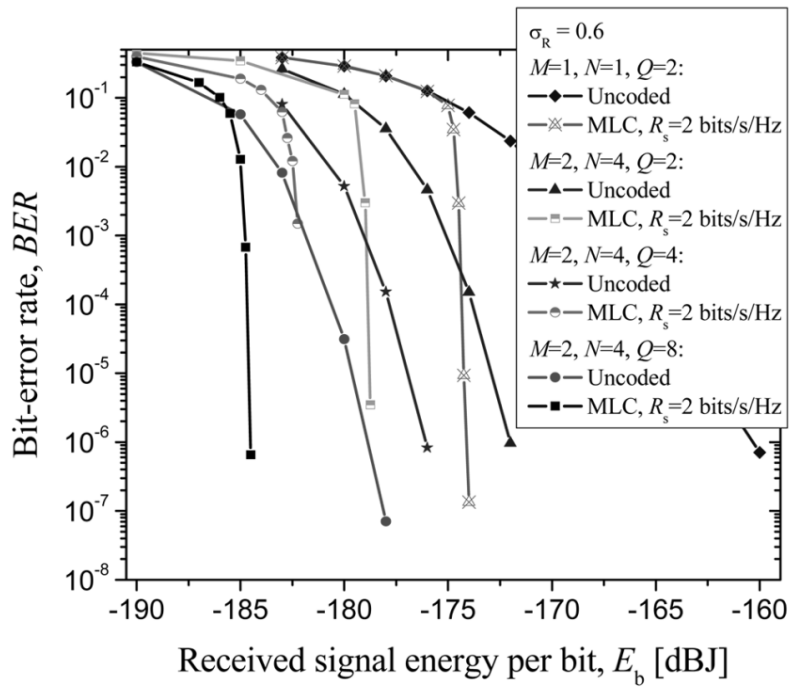

(a)

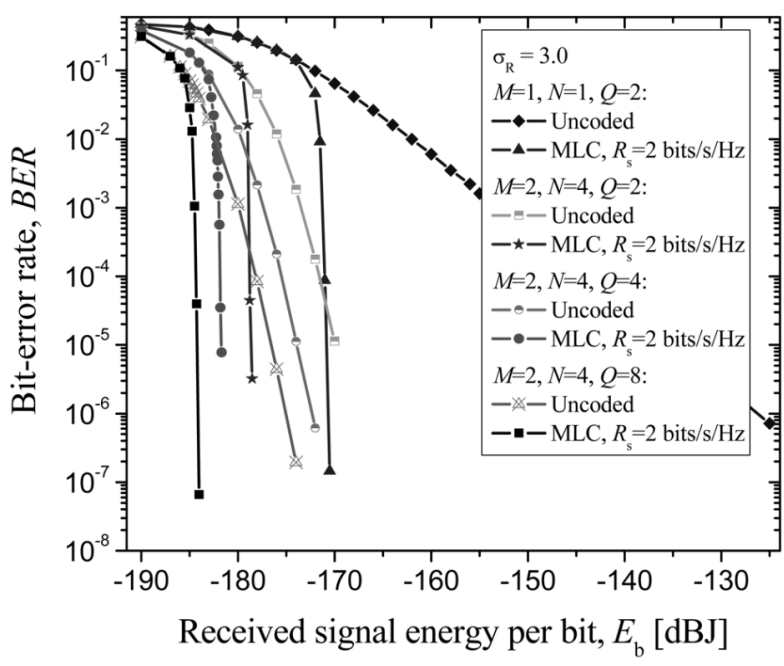

(b)

Fig. 3. BER performance of MLC for FSO MIMO systems with $Q$-ary PPM under (a) weak turbulence $\left(\sigma_{R}=0.6\right)$ and (b) strong turbulence $\left(\sigma_{R}=3\right)$. (Color version available online at http://ieeexplore.ieee.org.)

where $I$ is the signal intensity, $\Gamma(\cdot)$ is the gamma function, and $K_{\alpha-\beta}(\cdot)$ is the modified Bessel function of the second kind and order $\alpha-\beta$. The PDF parameters $\alpha$ and $\beta$, defined in [10], represent the effective number of large-scale and small-scale cells for zero-inner scale, respectively [4], and are defined as a function of Rytov variance $\sigma_{R}^{2}$, given by

$$
\sigma_{R}^{2}=1.23 C_{n}^{2} k^{7 / 6} L^{11 / 6} .
$$

$k=2 \pi / \lambda$ is the optical wave number, $L$ is propagation distance, and $C_{n}^{2}$ is the refractive index structure parameter, which we assume to be constant for horizontal paths.

\section{Performance Analysis And Conclusion}

The results of simulations are given in Fig. 3(a) for the weak turbulence and 3(b) for the strong turbulence. The symbol energy due to background light is set to $-170 \mathrm{dBJ}$. The MLC/PID scheme based on block-circulant LDPC codes with 2 bits/symbol is employed. For 8-PPM transmission, the MLC scheme supports up to three RF channels. The first data stream is encoded using $\operatorname{LDPC}(4320,2160)$ code, the second using
$\operatorname{LDPC}(4320,3242)$, and the third using $\operatorname{LDPC}(4320,3242)$ code. BER improves as the number of lasers and photodetectors increases. In the presence of background light, the BER decreases as the number of slots in $Q$-ary PPM scheme increases. The proposed MLC scheme provides excellent coding gains for different turbulence conditions, ranging from the weak to the strong turbulence regimes. For example, under the weak turbulence regime $\left(\sigma_{R}=0.6\right)$ the FSO repetition MIMO system with $M=2, N=4, Q=8$ supplemented with MLC with $2.0 \mathrm{bits} / \mathrm{symbol}$ provides the $24.25 \mathrm{-dB}$ improvement at BER of $10^{-6}$ over uncoded binary case with one laser and one PD $(M=1, N=1, Q=2)$. The MLC alone (for $M=1, N=1, Q=2)$ provides the coding gain of $13.86 \mathrm{~dB}$ at the same BER. Overall improvement of $24.25 \mathrm{~dB}$ can be split as follows: $11.76 \mathrm{~dB}$ improvement due to repetition MIMO processing $(M=2, N=4), 6.84 \mathrm{~dB}$ due to increase in number of slots $(Q=8)$, and $5.65 \mathrm{~dB}$ due to coding. The largest coding gain is obtained for $M=1, N=1, Q=2$. Under the strong turbulence regime $\left(\sigma_{R}=3.0\right)$ the same coded modulation scheme provides the improvement of $57.8 \mathrm{~dB}$ at BER of $10^{-6}$. The MLC scheme itself (for $M=1, N=1, Q=2$ ) provides the coding gain $44.3 \mathrm{~dB}$ at BER of $10^{-6}$. The simulations are performed for up to ten iterations in LDPC decoder.

The results reported in Fig. 3 are given in terms of energy per bit and are bit-rate invariant. The reason lies in the fact that the system is not modeled as the additive white Gaussian channel and because an ideal photon counting receiver is observed. The energy per bit is the same for different symbol durations and average powers, as given in (4). For more details on the bitrate dependency, the interested reader is referred to an excellent paper by Wilson et al. [2, Appendix].

\section{REFERENCES}

[1] X. Zhu and J.M. Kahn, "Free-space optical communication through atmospheric turbulence channels," IEEE Trans. Commun., vol. 50, no. 8, pp. 1293-1300, Aug. 2002.

[2] S. G. Wilson, M. Brandt-Pearce, Q. Cao, and J. J. H. Leveque III, "Free-space optical MIMO transmission with $Q$-ary PPM," IEEE Trans. Commun., vol. 53, no. 8, pp. 1402-1412, Aug. 2005.

[3] S. G. Wilson, M. Brandt-Pearce, Q. Cao, and M. Baedke, "Optical repetition MIMO transmission with multipulse PPM," IEEE Sel. Areas Commun., vol. 23, no. 9, pp. 1901-1910, Sep. 2005.

[4] M. A. Al-Habash, L. C. Andrews, and R. L. Phillips, "Mathematical model for the irradiance probability density function of a laser beam propagating through turbulent media," Opt. Eng., vol. 40, pp. 1554-1562, 2001.

[5] H. Park and J. R. Barry, "Trellis-coded multiple-pulse-position modulation for wireless infrared communications," IEEE Trans. Commun., vol. 52, no. 4, pp. 643-651, Apr. 2004.

[6] R. McEliece, "Practical codes for photon communication," IEEE Trans. Inf. Theory, vol. IT-27, no. 4, pp. 393-397, Jul. 1981.

[7] D. Fares, "Performance of convolutional codes with multipulse signaling in optical channels," Microw. Opt. Technol. Lett., vol. 3, pp. 406-410, Nov. 1990.

[8] S. S. Muhammad, E. Leitgeb, and O. Koudelkat, "Multilevel modulation and channel codes for terrestrial FSO links," in Proc. 2nd Int. Symp. Wireless Communication Systems, 2005, pp. 795-799.

[9] H. Imai and S. Hirakawa, "A new multilevel coding method using error correcting codes," IEEE Trans. Inf. Theory, vol. IT-23, no. 5, pp. 371-377, May 1977.

[10] J. A. Anguita, I. B. Djordjevic, M. A. Neifeld, and B. V. Vasic, "Shannon capacities and error-correction codes for optical atmospheric turbulent channels," OSA J. Opt. Networking, vol. 4, pp. 586-601, Sep. 2005.

[11] J. Hou, P. H. Siegel, L. B. Milstein, and H. D. Pfitser, "Capacityapproaching bandwidth-efficient coded modulation schemes based on low-density parity-check codes," IEEE Trans. Info. Theory, vol. 49, no. 9, pp. 2141-2155, Sep. 2003. 